\title{
Shaping proto-planetary nebulae by binary systems
}

\author{
A. Riera ${ }^{1}$, P. F. Velázquez ${ }^{2}$, W. Steffen ${ }^{3}$, A. C. Raga ${ }^{2}$ and J. Cantó ${ }^{4}$ \\ ${ }^{1}$ Departament de Física i Enginyeria Nuclear, EUETIB, Universitat Politécnica de Catalunya \\ Compte d'Urgell 187, 08036 Barcelona, Spain \\ email: angels.riera@upc.edu \\ ${ }^{2}$ Instituto de Ciencias Nucleares, Universidad Nacional Autónoma de México, México D.F., \\ México \\ ${ }^{3}$ Instituto de Astronomía, Universidad Nacional Autónoma de México, Ensenada, México \\ ${ }^{4}$ Instituto de Astronomía, Universidad Nacional Autónoma de México \\ Apdo. Postal 70-242, CP: 04510, D.F., México
}

\begin{abstract}
We present the results of 3D hydrodynamic simulations aimed to explore the binary scenario for shaping bipolar, point- and mirror-symmetric proto-Planetary Nebulae. We consider a jet launched by the secondary star of a binary system, located at the center of the PPN, which propagates within a circumstellar medium swept previously up by the wind of the giant companion. As a result of the presence of the companion star, the accretion disk around the jet source is likely to precess. We have carried out $3 \mathrm{D}$ hydrodynamical simulations with the YGUAZÚ-A code including the combination of an orbital motion plus a precession. Our results show that to produce a multipolar nebula, it is necessary to have a precessing jet in a binary system with a time-dependent ejection velocity.
\end{abstract}

Keywords. Planetary nebulae: general, ISM: jets and outflows

\section{Introduction}

Proto-PNe (PPNe) and young PNe often exhibit a very rich morphology with strong deviations from spherical symmetry. Several classification schemes have been presented in the past. The first PNe classification were based on the large-scale structure (Manchado et al. 2000, Stanghellini et al. 1993, and Stanghellini et al. 1999). Several PNe and PPNe imaging surveys have been carried out with the HST which reveal new structures. Sahai et al. $(2007,2011)$ from the analysis of the HST/WFPC2 images they postulated four major classes: bipolar (B), multipolar (M), elliptical (E) and irregular (I). The bipolar nebulae have two primary, diametrically opposed lobes and represent the $\sim 30 \%$ of their sample, the multipolar nebulae show two or more primary lobe pairs whose axis are not aligned (20\% of the sample). Preliminary results of imaging surveys of postAGB stars have been presented in this conference. The mid-infrared survey conducted by Lagadec et al. (these proceedings) reveals that $20 \%$ of the resolved objects shows B morphology and $7 \%$ displays $\mathrm{M}$ geometry. A somehow larger fraction of $\mathrm{B}$ and $\mathrm{M}$ nebulae are found within a sample of 140 post-AGB objects analyzed by Manchado et al. (these proceedings).

The existence of a binary system has been invoked as the main mechanism to shape $\mathrm{B} / \mathrm{M}$ nebulae. This suggestion is supported by the increasing number of B/M nebulae that host a central binay system. Recently, Miszalski et al. (2009) estimated a binary fraction of $(17 \pm 5) \%$ for their PNe sample. This fraction seems low in comparison with the large fraction of PNe with B or M morphology. However, it has to be considered as a lower limit because this survey cannot detect large orbital periods. 
The aim of this work is to explore (3D gasdynamical simulations) the influence of having a binary system in order to reproduce a bipolar or multipolar nebula.

\section{Binary system scenario}

We consider a jet from a source in orbital motion around a binary companion (see Figure 1 of Haro-Corzo et al. 2009). One could have a binary system formed by a low-mass main sequence star (the source of the jet), and a more massive red giant star. As the red giant star expands, it overflows its Roche lobe, producing a stream of material that falls towards the binary companion, which forms an accretion disk around the main sequence star. Part of the material that is being accreted is ejected as a bipolar outflow. We assume that the bipolar flow is always perpendicular to the disk. Therefore if the disk precesses, this precession will also be present in the ejection axis.

Models of accretion disks around a star in a binary system predict that the disk will have a retrograde precession (Terquem et al. 1999). Employing figure 1 of Raga et al. (2009) and assuming $M_{1} / M_{2} \leqslant 10$, we find that the ratio between the precession and orbital periods $\tau_{p} / \tau_{o} \geqslant 2$. The jet/counterjet flow in a binary system with a precessing disk, will be affected by both the precession and the orbital motion.

The effect of a full precession period will be seen over a distance

$$
D_{p}=v_{j} \tau_{p}
$$

where $v_{j}$ is the jet velocity and $\tau_{p}$ the precession period. This would correspond to a full loop of a precession spiral with half-opening angle $\alpha$, which is a free parameter.

Structures associated with the orbital motion have typical sizes given by:

$$
D_{o}=v_{j} \tau_{o},
$$

where $\tau_{o}$ is the orbital period. The spiral resulting from the orbital motion has mirror symmetry between the jet and the counterjet lobes, as opposed to the point symmetry of the precession spiral. From the disk precession models, we expect $\tau_{p} \sim 2 \rightarrow 20 \tau_{o}$. This implies that the loops of the precession spiral will have length scales $\sim 2 \rightarrow 20$ times longer than the size of the loops due to the orbital motion. Therefore, we would expect the jet/counterjet system to have a mirror symmetric spiral shape close to the source and a transition to a broader, point-symmetric precession spiral at larger distance from the source (Raga et al. 2009).

In the following, we present 3D numerical simulations performed with the YGUAZÚ-A hydrodynamical code (Raga et al. 2000). In these simulations, we assume that the jet is launched from the companion star.

\section{The Red Rectangle: the result of a precessing jet?}

In specific objects the effect of precession or the effect of the orbital object might not be present. For example, we could have a low orbital motion in which case the opening angle of the spiral induced by the orbital motion would be very small. This seems to be the case of the Red Rectangle (Velázquez et al. 2011).

The Red Rectangle is a PPN associated with the binary system HD44179 which has a $\tau_{o} \sim 320$ days (Men'shchikvov et al. 2002). This PPN has a striking biconical morphology with the presence of the so-called ladder rungs, which are linear features almost perpendicular to the nebula symmetry axis, and a pattern of nested parabola in the 

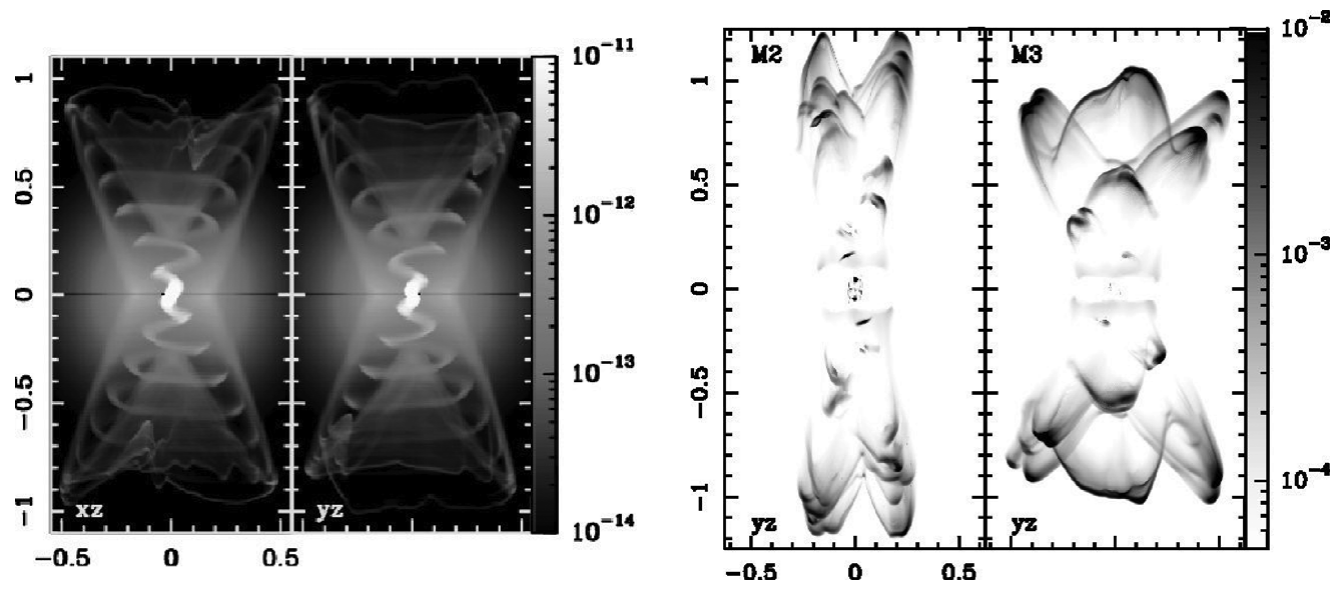

Figure 1. Left: Synthetic scattered light intensity maps obtained for $t=200$ yr suited to reproduce the morphology of the Red Rectangle, considering an inclination of the line of sight with the y-axis (xz-projection, left) or the x-axis (yz-projection, right) of $10^{\circ}$. Right: Synthetic $\mathrm{H} \alpha$ emission maps (yz-projection) for models M2 and M (see text). The vertical and horizontal axis are in units of $10^{17} \mathrm{~cm}$.

ERE (continuous extended red emission) (Cohen et al. 2004). In the scattered light the nebula is approximately spherical. The "ladder rungs" could be the result of the precession and/or orbital motion. Employing the Fig. 1 of Raga et al. (2009), we deduced that $\tau_{p} \sim 10 \tau_{o}$. Velázquez et al. (2011) deduced that the large scale morphology could be produced by the precession motion alone. For this reason, we have neglected the effect of the orbital motion in the model of the Red Rectangle.

We carried out a 3D numerical simulation suited to model the Red Rectangle nebula (see details in Velázquez et al. 2011). The jet/counter jet system is injected at the centre of the computational domain and evolves into a circumstellar medium previously shaped by the slow wind from the AGB star. The axis of the jet precesses describing a cone with a semiaperture angle $\alpha=30^{\circ}$ and a precession period of $17.6 \mathrm{yr}$. The jet velocity $v_{j}$ and its number density $n_{j}$ were set to $300 \mathrm{~km} \mathrm{~s}^{-1}$ and $5 x 10^{4} \mathrm{~cm}^{-3}$, respectively. In order to compare the hydrodynamical results with the observation of the Red Rectangle, we have generated scattered light intensity maps (shown in 1). The synthetic maps show an $\mathrm{X}$-shaped structure which correspond to the walls of the cocoon produced by the jets. As the jet evolves around the precession axis both the jet and the cocoon describe a helical path (see 1). This helical path looks almost horizontal and can be associated with the "ladder rungs".

\section{Multipolar PPNe}

As previously shown, bipolar PPNe, either with point- or mirror symmetry, can be associated with precession and/or orbital motion of the jet source (Haro-Corzo et al. 2009; Velázquez et al. 2011). However, we have found that to form multipolar lobes, it is necessary to introduce a variability in the jet velocity. We assume that the velocity variability period is equal to the orbital one. If the orbit is elliptical, we expect that the accretion, and also the jet launching, to be more efficient when the stars pass through the periastron. 
As a first approach, we will associate the typical sizes of the lobes of M nebulae $\left(D_{l}\right.$ of the order of few $10^{17} \mathrm{~cm}$ ) with the precession motion and obtain a characteristic time:

$$
\tau=\frac{D_{l}}{v_{j} \cos \alpha}=p \tau_{p}
$$

where $\alpha$ is the semi-aperture angle of the precession cone. This characteristic time can be written as a factor $p$ times the precession period $\tau_{p}$. From the work of Terquem et al. (1999), $\tau_{p} / \tau_{o}$ is a function of the ratio $m_{2} / m_{1}$, being $m_{2}$ and $m_{1}$ the stellar masses of the components of the central binary system. If we consider $\tau_{p}=q \tau_{o}$, it would be possible to get an estimate for $\tau_{o}$ from Eq. (4.1):

$$
\tau_{o}=\frac{D_{l}}{p q v_{j} \cos \alpha},
$$

Eq.(4.2) tells us that from observational arguments and choosing a value for the parameters $p$ and $q$, we can determine the orbital period of the central source.

We have computed several models with the aim to reproduce the multipolar geometry observed in several PPNe (details in Velázquez et al. submitted). Here, we present the results of two models (M2 and M3) that produce a four lobed (M2) and a six lobed morphology (M3). The main velocity of the jet $v_{j}$ of $250 \mathrm{~km} \mathrm{~s}^{-1}$, a number density $n_{j}$ of $5 \times 10^{4} \mathrm{~cm}^{-3}$, an orbital eccentricity $\epsilon=0.5$, and $p=2$. Both models have a sinusoidal velocity variability with an amplitude $\Delta \mathrm{v}$ of $75 \mathrm{~km} \mathrm{~s}^{-1}$. The semi-aperture angles of the precession cone $(\alpha)$ were set to $15^{\circ}(\mathrm{M} 2)$ and $30^{\circ}$ (M3).

In the case of model M2, we adopted a value of 4 for the $q$ parameter and at $t=0$ we set the jet direction to form an initial angle $\left(\phi_{p o}\right)$ of $\pi / 4$ with respect to the $\mathrm{x}$-axis. As a result, the predicted $\mathrm{H} \alpha$ emission map show four lobes (see Figure1). The $\mathrm{H} \alpha$ intensity map derived for model M3 shows six lobes. In the latter case, $q=0$ and $\phi_{p o}=0$ (see Figure1).

\section{References}

Cohen, M., Van Winckel, H., Bond, H. E., \& Gull, T. R. 2004, AJ, 127, 2362

Haro-Corzo, S. A. R., Velázquez, P. F., Raga, A. C., Riera, A., \& Kajdic, P. 2009, ApJ (Letters), 703, L18

Miszalski, B., Acker, A., Moffat, A. F. J., Parker, Q. A., \& Udalski, A. 2009, A $\& A, 496,813$

Manchado, A., Villaver, E., Stanghellini, L., \& Guerrero, M. A. 2000, in: J.H. Kastner, N. Soker \& S. Rappaport, Asymmetrical Planetary Nebulae II: From Origins to Microstructures, ASP Conference Series, 199, p. 17

Men'shchikov, A. B., Schertl, D., Tuthill, P. G., Weigelt, G., \& Yungelson, L. R. 2002, A\&্A, 393, 175

Raga, A. C., Navarro-González, R., \& Villagrán-Muniz, M. 2000, Rev. Mexicana AyA, 36, 67

Raga, A. C., Esquivel, A., Velázquez, P. F., Cantó, J., Haro-Corzo, S., Riera, A., \& RodríguezGonzález, A. 2009, ApJ (Letters), 707, L6

Sahai, R., Morris, M., Sánchez Contreras, C., \& Claussen, M. 2007, AJ, 134, 2200

Sahai, R., Morris, M., \& Villar, G. G. 2011, AJ, 141, 134

Stanghellini, L., Blades, J. C., Osmer, S. J., Barlow, M. J., \& Liu, X.-W. 1999, ApJ, 510, 687

Stanghellini, L., Corradi, R. L. M., \& Schwarz, H. E. 1993, A\& A, 276, 463

Terquem, C., Eislöffel, J., Papaloizou, J. C. B., \& Nelson, R. P. 1999, ApJ (Letters), 512, L131

Velázquez, P. F., Steffen, W., Raga, A. C., Haro-Corzo, S., Esquivel, A., Cantó, J., \& Riera, A. 2011, ApJ, 734, 57 